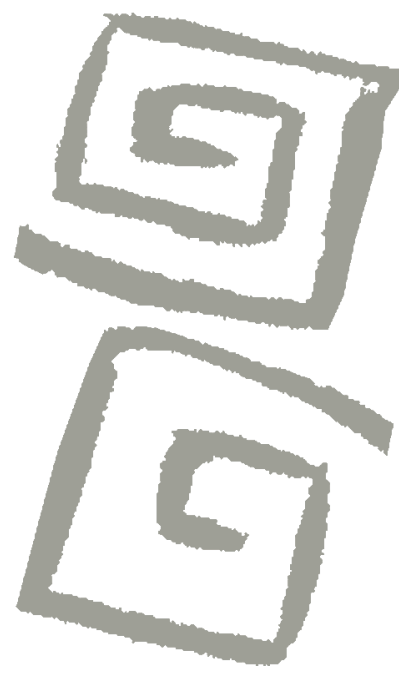

\title{
Habitus alimentario: prácticas entre trabajadores agrícolas migrantes en una comunidad de Sonora, México
}

\author{
Food habitus: practices among migrant farm workers in \\ a community of Sonora, Mexico
}

María del Carmen Arellano Gálvez', Guadalupe del Carmen Alvarez Gordillo², Enrique Eroza Solana ${ }^{3}$, Laura Huicochea Gómez ${ }^{4}$ Esperanza Tuñón Pablos ${ }^{5}$

${ }^{1}$ Doctora en Ciencias en Ecología y Desarrollo Sustentable, El Colegio de la Frontera Sur, México. $\triangle$ iD

${ }^{2}$ Doctora en Ciencias Biológicas y de la Salud. Profesora Investigadora, El Colegio de la Frontera Sur, México. $\triangle$ iD

${ }^{3}$ Doctor en Filosofía. Profesor Investigador Centro de Investigación y Estudios Superiores en Antropología Social, México. $\triangle$ iD

${ }^{4}$ Doctora en Antropología. Profesora Investigadora, EI Colegio de la Frontera Sur, México. $\triangle$ (iD)

${ }^{5}$ Doctora en Sociología. Profesora Investigadora, EI Colegio de la Frontera Sur, México. $\triangle$ iD
RESUMEN El objetivo es analizar el habitus alimentario y los cambios en las prácticas de alimentación, comparando trabajadores agrícolas migrantes pendulares y asentados en la ciudad de Miguel Alemán, del estado de Sonora. A partir de un enfoque etnográfico, se realizaron 21 entrevistas en profundidad entre ambos tipos de migrantes desde enero de 2016 hasta enero de 2018. Encontramos que, en ambos tipos de migrantes, aumenta el consumo de productos industrializados, como harinas y azúcares, cuyos efectos en la salud provocan malnutrición, principalmente en migrantes pendulares, y enfermedades crónicas como diabetes, en migrantes asentados. Concluimos que el habitus alimentario se ajusta a la disponibilidad física y económica de los alimentos y la lógica del trabajo agrícola, así la alimentación se estructura como una práctica por necesidad. Esto reproduce material y simbólicamente la posición subordinada de los trabajadores agrícolas frente al modelo alimentario y económico globalizado, cuyas prácticas de alimentación se recrudecen por las dificultades de acceso a los alimentos.

PALABRAS CLAVES Alimentación; Agricultura; Migración; Enfermedades Crónicas; México.

\begin{abstract}
The objective is to analyze the food habitus and the changes in eating practices of pendulum and settled migrant agricultural workers in the city of Miguel Aleman in the state of Sonora. Based on an ethnographic approach, 21 in-depth interviews were conducted with both types of migrants from January 2016 to January 2018 . We found that in these migrants, the consumption of industrialized products such as flours and sugars has increased, leading to malnutrition mainly in pendulum migrants and chronic diseases such as diabetes in settled migrants. We conclude that the food habitus is adjusted to the physical and economic availability of food and the logic of agricultural work, with eating structured as a practice of necessity. This reality materially and symbolically reproduces the subordinate position of agricultural workers in the face of the globalized economic and food production models, in which eating practices are debilitated by the difficulties of access to food.
\end{abstract}

KEY WORDS Feeding; Agriculture; Migration; Chronic Diseases; Mexico. 


\section{INTRODUCCIÓN}

Acabar con el hambre es el segundo de los 17 Objetivos de Desarrollo Sostenible (ODS) para el año 2030, emitidos por la Organización de las Naciones Unidas(1) luego de no alcanzar la meta de erradicar el hambre en el mundo, planteado por los Objetivos del Milenio (ODM). Este objetivo se vincula directamente con dos más: el fin de la pobreza y el procurar salud y bienestar para la población, temas prioritarios para el desarrollo sostenible. En la actualidad se produce alimento suficiente, sin embargo, campesinos, mujeres y niños de zonas rurales de países en vías de desarrollo viven con hambre ${ }^{(2)}$. La carencia alimentaria entre estos grupos vulnerados indica la pertinencia de abordar los habitus y prácticas alimentarias que se expresan en hambre, malnutrición y enfermedades crónicas como diabetes y obesidad, problemas actuales de salud pública recrudecidos en poblaciones con dificultades para acceder a los servicios de atención a la salud.

En este artículo mostramos cómo se relacionan procesos macroestructurales como la migración y el trabajo en la configuración del habitus alimentario, que analizaremos por medio de las prácticas de alimentación entre población vulnerada en México como son las y los trabajadores agrícolas, migrantes internos que se movilizan por distintos motivos, siendo uno de ellos la carencia de ingresos y de trabajo remunerado en sus lugares de origen. Provienen del sur-sureste de México, de estados como Chiapas, Veracruz, Oaxaca y Guerrero que tienen altos índices de marginación social ${ }^{(3)}$ y migran al norte a los estados de Sinaloa, Sonora y Baja California, donde se requiere de mano de obra para la producción de alimentos de exportación ${ }^{(4)}$. Gracias a su trabajo se distribuyen alimentos alrededor del mundo, generando ganancias a la agroindustria; sin embargo, viven en condiciones de pobreza e inseguridad alimentaria ${ }^{(5)}$, que se traducen en enfermedades como obesidad y desnutrición ${ }^{(6,7,8)}$.

A esto se suman las dificultades de acceso a los servicios de salud a través de su condición de trabajadores asalariados ${ }^{(9)}$, debido a políticas laborales que recrudecen la posibilidad de acceder y ejercer el derecho a la protección social y de salud ${ }^{(10,11,12)}$. Ante esta situación, la población acude a servicios otorgados por el Estado a población sin seguridad, lo que genera altos costos al sistema de salud mexicano y resulta insuficiente para atender enfermedades crónicas relacionadas con la alimentación y sus complicaciones ${ }^{(13,14,15)}$.

El objetivo es analizar el habitus alimentario y los cambios en las prácticas de alimentación entre trabajadores agrícolas migrantes pendulares y asentados en la ciudad de Miguel Alemán, del estado de Sonora. Es un trabajo cualitativo que analizamos a partir del habitus, para lo cual haremos referencia a las prácticas ${ }^{(16,17)}$, en este caso, las de alimentación, entendidas no como una decisión individual, sino como parte de procesos estructurales que ajustan las disposiciones a la acción de los agentes a través del habitus. Por esta razón haremos referencia a las prácticas que dan sentido al habitus alimentario entre ambos tipos de migrantes.

El artículo busca contribuir al análisis de la alimentación desde esta postura teórica de reciente aplicación en la investigación latinoamericana. Este artículo deriva de una investigación titulada "Relaciones sociales y prácticas de alimentación de migrantes del sureste mexicano, residentes en una comunidad agroindustrial de Sonora", de la cual se han derivado otros dos artículos: uno, ya publicado, que analiza las prácticas de alimentación desde la perspectiva de género ${ }^{(18)}$ y otro, aceptado para publicación, que analiza el campo alimentario.

\section{Contexto de estudio}

La agricultura es la principal actividad económica de Miguel Alemán, perteneciente al municipio de Hermosillo, Sonora. Desde 1990, se han realizado gestiones para convertir la ciudad en municipio, sin que a la fecha se haya concretado este proceso. Cuenta con una superficie cultivada de 41.034 hectáreas en, aproximadamente, 205 campos agrícolas, 
en donde se desarrolla agricultura de temporal y de riego altamente tecnificado ${ }^{(19)}$. Se siembran hortalizas, forrajes y productos frutícolas (uva, nuez y naranja principalmente), que se comercializan a EEUU, Canadá y China $^{(20,21)}$.

La localidad tiene una población de 40.000 habitantes $^{(19)}$ y su crecimiento se relaciona con el asentamiento de trabajadores agrícolas provenientes de estados del sur-sureste del país como Oaxaca, Guerrero, Chiapas, Veracruz y Puebla, y se estima que el $34 \%$ de la población nació en otro estado. Por los altos índices de marginación, la localidad fue incluida en 2013 como una de las zonas prioritarias del programa federal Cruzada contra el Hambre y, desde finales de 2016, opera el primer comedor comunitario ${ }^{(22)}$. El gobierno municipal 2015-2018 diseñó un programa de desarrollo urbano para la regularización de asentamientos y el acceso a servicios como agua, alumbrado público y pavimentación.

Miguel Alemán cuenta con dos centros de salud y una clínica del Instituto Mexicano del Seguro Social (IMSS); el 67,3\% de la población está adscrita a algún servicio de salud, el 36,4\% tiene Seguro Popular (póliza para proteger a población sin seguro social) y el $30,9 \%$ está adscrito al IMSS, ISSSTE e ISSSTESON (Instituto de Seguridad Social para Trabajadores del Estado a nivel federal y estatal). El porcentaje de la población sin ningún servicio médico es del $32,2 \%{ }^{(19)}$.

La Jurisdicción Sanitaria I reportó que, en 2009, las complicaciones por diabetes mellitus fueron la principal causa de mortalidad en Miguel Alemán. Investigaciones refieren desnutrición, sobrepeso y obesidad en población migrante ${ }^{(7,8,23)}$, la relación entre diabetes y prácticas de alimentación ${ }^{(24)}$, así como inseguridad alimentaria entre población asentada y aumento de obesidad, principalmente, entre mujeres ${ }^{(6)}$. Analizar el habitus alimentario permitirá comprender el sentido lógico de las prácticas, y evitar culpabilizar y/o estigmatizar a la población que vive cotidianamente la violencia estructural ${ }^{(25)}$ que la subordina en distintos campos, incluyendo la salud y la alimentación.

\section{Marco analítico}

Para Bourdieu ${ }^{(16)}$ y Bourdieu y Wacquant ${ }^{(17)}$, el habitus se refiere al sentido lógico de las prácticas que producen y reproducen un orden social que se expresa en disposiciones a la acción, en principios generadores y organizadores de las prácticas construidas socialmente. El habitus se refiere a "sistemas perdurables y trasladables de esquemas de percepción, apreciación y acción que resultan de la institución de lo social en el cuerpo" $^{\prime \prime(17)}$. Se trata de disposiciones abiertas a experiencias que modifican o refuerzan las estructuras en las que se producen. El habitus es duradero pero no inmutable, es lo social hecho cuerpo, dando sentido lógico a las prácticas, es lo internalizado de las oportunidades objetivas y de las condiciones sociales y económicas. A través de él se genera el sentido común, las regularidades conductuales y de pensamiento que se corresponden a las condiciones objetivas del campo, una especie de libertad controlada.

El habitus se significa solo en relación con el campo, entendido como una red de relaciones objetivas entre las distintas posiciones que ocupan los agentes en la estructura y distribución del poder, produciendo y reproduciendo relaciones de dominación y subordinación. Pero los agentes no responden mecánicamente a la estructura, de ahí que el habitus no es destino, sino un sistema de disposiciones estratégicas que funcionan en y para la práctica ${ }^{(17)}$.

Los agentes se ubican en distintas posiciones de poder en los campos sociales, que definen lo accesible y lo inaccesible, lo posible y lo imposible, por lo que las prácticas se ajustan a las condiciones objetivas para satisfacer deseos o necesidades al sentido lógico, a través de esquemas con los que se percibe el mundo, se valora lo bueno y lo malo y se aprecian los gustos y los disgustos ${ }^{(26)}$. El gusto por los alimentos se relaciona con el lugar que ocupan los agentes en los campos sociales, por lo que para el análisis de las preferencias alimenticias es necesario considerar las condiciones de posibilidad objetivas, comprendidas como los elementos estructurales 
y materiales de vida que dan sentido lógico a la práctica de los agentes sociales, así como el capital económico, social y cultural ${ }^{(16)}$.

Bourdieu $^{(27)}$ refiere que los gustos y preferencias alimentarias tienen un origen social diferenciado entre clases sociales como signo de distinción, no es una decisión individual sino que está relacionada con la posición de los agentes en los campos sociales. El autor refiere que, entre los agentes de clases sociales bajas, hay preferencia por alimentos con alto contenido energético, en los que invierten la mayoría de su capital económico. Los gustos alimenticios se relacionan con las condiciones económicas en las que se produce el habitus, por lo que para su análisis se consideran las relaciones de dominación entre las clases y solo con base en este contraste se comprenden las diferencias entre los gustos. Entre grupos poblacionales en condiciones de precariedad económica, "la necesidad impone un gusto por necesidad que implica una forma de adaptación a la necesidad, y con ello, de aceptación de lo necesario, de resignación a lo inevitable"(27).

La elección por lo necesario se basa en aquello que es práctico y funcional en relación con las condiciones objetivas de existencia, mientras que se renuncia a otras prácticas inaccesibles, aquella que "no es para noso-

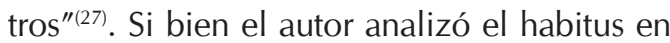
clases sociales de Francia en la década de 1980, en este artículo no utilizamos la clase social como categoría analítica ya que rebasa su objetivo. Por esta razón haremos referencia a las trabajadoras y los trabajadores agrícolas como grupo poblacional que comparten una posición subordinada económica, política, social e históricamente. En el texto comprendemos el habitus alimentario como los esquemas de percepción y apreciación que disponen a prácticas relacionadas con la alimentación. A partir de esta perspectiva, analizamos el habitus alimentario y los cambios en las prácticas de alimentación relacionadas con la experiencia migratoria de un grupo poblacional que encuentra, en el trabajo agrícola, las condiciones de posibilidad para adquirir el capital económico para sobrevivir, a pesar de tener varias carencias, incluyendo la alimentación ${ }^{(4)}$.

\section{METODOLOGÍA}

Esta investigación etnográfica fue realizada en distintos periodos desde enero de 2016 a enero de 2018, en Miguel Alemán, Sonora, mediante entrevistas en profundidad a trabajadores agrícolas asentados y pendulares, y observación participante y no participante en los hogares y espacios públicos de la localidad. Por migrantes asentados, comprendemos a aquellos que tienen más de cuatro años de residir en la comunidad, mientras que, los migrantes pendulares, son aquellos que tienen menos de este tiempo de residir en el lugar, ya que se movilizan por los diversos estados en donde se desarrolla la agroindustria y luego retornan a sus comunidades $^{(28)}$. Estas categorías se han analizado en estudios sobre migración interna en México para mostrar los circuitos migratorios, los procesos de asentamiento en las localidades de destino y el despoblamiento de las localidades de origen ${ }^{(29)}$.

La entrevista permite obtener información sobre un tema en particular, a través de los discursos, experiencias y narrativas de las y los agentes entrevistados a partir de preguntas abiertas ${ }^{(30,31)}$. La entrevista en profundidad requiere una relación de confianza para llevar una conversación fluida y natural que parta de asuntos generales para después profundizar. Se replantean preguntas para corroborar información y llegar al punto de saturación teórica, es decir, cuando ya no se generan apreciaciones adicionales a lo escuchado, analizado y observado ${ }^{(31,32,33)}$. A través de las entrevistas indagamos sobre las prácticas alimentarias en los lugares de origen, los cambios que se dan durante los tránsitos y el asentamiento, así como las dificultades y posibilidades para acceder a los alimentos.

Se incluyeron hombres y mujeres con distinto tiempo de residencia en la localidad, que hayan tenido experiencia previa de trabajo agrícola y que fueran originarios de estados del sur-sureste mexicano. La observación participante se realizó en los hogares, participando en la elaboración de comidas, apoyando en el riego de los cultivos de 
traspatio y acompañando a las personas al supermercado. También se acudió a tiendas de abarrotes, tianguis, puestos de comidas y taquerías.

Se realizaron 21 entrevistas en profundidad, 11 a migrantes pendulares y 10 a asentados en Miguel Alemán. Todas las personas entrevistadas, alguna vez en su trayecto migratorio, trabajaron en los campos agrícolas y algunas se han insertado en otras actividades productivas, como la venta de comidas, bebidas y el cuidado de menores. Del total, 11 participantes eran mujeres y 10 , hombres, cuya edad osciló entre los 18 y los 74 años. Respecto al lugar de origen, una es de Puebla, dos de Oaxaca, cuatro de Veracruz, siete de Chiapas y siete de Guerrero (Tabla 1).
Se obtuvo la aprobación del protocolo de investigación por parte del Comité de Ética en Investigación de El Colegio de la Frontera Sur (CEI-O-077/16, aprobado el 12 de febrero de 2016), así como el consentimiento informado de los participantes, a quienes se les asignaron pseudónimos para proteger su confidencialidad. Se reiteró el derecho a declinar su participación y/o a no responder. Algunas entrevistas fueron grabadas y se transcribieron en su totalidad; en otras entrevistas y observaciones se tomaron notas y se transcribieron en el diario de campo. La información empírica fue analizada y codificada utilizando el programa Nvivo versión 10. Del cúmulo de información, se retoma aquella que nos permite analizar el habitus alimentario.

Tabla 1. Datos sociodemográficos de participantes en entrevistas en profundidad. Miguel Alemán, Sonora, México. 2016-2018.

\begin{tabular}{|c|c|c|c|c|c|}
\hline $\begin{array}{l}\text { Tipo de } \\
\text { migración }\end{array}$ & Pseudónimo & Edad & $\begin{array}{l}\text { Lugar de } \\
\text { origen }\end{array}$ & Escolaridad & $\begin{array}{l}\text { Tiempo de residir en } \\
\text { Miguel Alemán }\end{array}$ \\
\hline \multirow{10}{*}{$\begin{array}{l}\text { Migrantes } \\
\text { asentados }\end{array}$} & Jorge & 18 & Oaxaca & Secundaria inconclusa & 4 años \\
\hline & Mónica & 24 & Guerrero & Preparatoria inconclusa & 5 años \\
\hline & Celia & 34 & Oaxaca & Secundaria & 14 años \\
\hline & Elena & 40 & Chiapas & Ninguna & 7 años \\
\hline & Angelina & 50 & Chiapas & Ninguna & 12 años \\
\hline & Arnoldo & 50 & Guerrero & Ninguna & 22 años \\
\hline & Teresa & 50 & Guerrero & Ninguna & 22 años \\
\hline & Isaura & 52 & Veracruz & Ninguna & 20 años \\
\hline & Esperanza & 73 & Chiapas & Ninguna & 20 años \\
\hline & Esteban & 74 & Chiapas & Ninguna & 20 años \\
\hline \multirow{11}{*}{$\begin{array}{l}\text { Migrantes } \\
\text { pendulares }\end{array}$} & Juan & 18 & Chiapas & Preparatoria inconclusa & 5 meses \\
\hline & Anselmo & 24 & Puebla & Primaria & 2 años \\
\hline & Lucía & 24 & Guerrero & ND & 3 meses \\
\hline & Luz & 24 & Chiapas & Preparatoria inconclusa & 1 año \\
\hline & Germán & 25 & Chiapas & Primaria & 2 años \\
\hline & José & 30 & Guerrero & ND & 3 meses \\
\hline & Isidro & 30 & Guerrero & Ninguna & 3 meses \\
\hline & Alma & 47 & Veracruz & Ninguna & 3 años \\
\hline & Eduardo & 56 & Guerrero & Preparatoria inconclusa & 6 meses \\
\hline & Sofía & ND & Veracruz & Ninguna & 9 meses \\
\hline & Bernardo & ND & Veracruz & Ninguna & 9 meses \\
\hline
\end{tabular}




\section{RESULTADOS}

Los resultados se analizan en dos apartados: 1) el habitus alimentario en el lugar de origen: prácticas recordadas y añoradas; y 2) habitus alimentario en el contexto de migración: prácticas y ajustes. En el primer apartado la información empírica es menor que la del segundo, esto permite contextualizar el cambio en las prácticas de alimentación, relacionadas con las formas de organización sociocultural y económica de las zonas de origen, así como el contexto medioambiental que posibilitaba ciertas prácticas.

\section{Habitus alimentario en el lugar de origen: prácticas recordadas y añoradas}

En los datos empíricos es necesario considerar la añoranza por los alimentos y cómo esta influye en la reconstrucción de las narrativas respecto a la posibilidad de haber padecido hambre en años pasados en los lugares de origen, donde la pobreza y marginación es común. Tales valoraciones sobre los alimentos se inscriben en esquemas de percepción y apreciación que, a través del tiempo, pueden idealizarse como parte de las nostalgias alimentarias. Tanto las y los migrantes que tienen más de cuatro años de asentamiento como aquellos de reciente Ilegada a Miguel Alemán provienen de localidades rurales que no han sido ajenas al proceso de globalización, ya que refieren en sus prácticas a una combinación de patrones modernos y tradicionales de alimentación. Sus testimonios indican la reproducción de prácticas de cultivo y cría de animales para consumo y venta en pequeña escala, en donde el acceso a frutas y verduras es variado, lo que es posibilitado por el medio geográfico-ambiental:

Traía mi papá pescados, mojarras, conejo, unas palomotas. Él iba al monte y traía quelites, hongos. Con lo poquito que trabajaba y lo que vendía de su cosecha, nos compraba ropa, zapatos y nos traía unas latotas de galletas. (Isaura, Veracruz, Asentada)

Isaura refiere prácticas de autoconsumo, incluyendo la pesca y la recolección, mezcladas con el consumo de productos procesados y envasados. Documentamos el consumo de distintas verduras, frutas y carne de animales silvestres como una práctica compartida en contextos donde la monetarización de la vida cotidiana era incipiente, dando lugar a prácticas como la donación, el intercambio o trueque y la venta a pequeña escala. Elena, quien tiene 12 años de asentamiento refiere:

\begin{abstract}
Antes comíamos con mi mamá casi pura verdura, casi no comía carne... no tenía mucho dinero para comprar cosas... pollo de rancho y algunas personas mataban chivos, borregos y nos invitaban. (Elena, Chiapas, Asentada)
\end{abstract}

También podemos analizar la valoración sociocultural de los alimentos de origen animal en detrimento de las prácticas de alimentación relacionadas con la dieta mesoamericana, en la cual la milpa tiene un papel central ${ }^{(34)}$. El acceso a alimentos por la siembra se complementaba con la compra de alimentos procesados, cuyo dinero provenía de la venta parcial de los cultivos, tal como Mónica refiere:

Comíamos quelite, ejote, para hervir en caldos, y del rio, pescadito, cangrejito, ranita y cosas que aquí no hay... venado, armadillo, iguana. Mi papá sembraba maíz, frijol, calabaza, jamaica. Engordaba sus animales y comía. El dinero era para una soda, azúcar. (Mónica, Guerrero, Asentada)

La diversidad de frutas, hortalizas y carnes referidas por las y los participantes no se encuentran en la zona de asentamiento, como veremos más adelante. También documentamos las prácticas de autoconsumo entre migrantes pendulares, cuya edad oscila entre los 18 y 56 años, indicando el proceso histórico de modernización de las localidades rurales en México. Eduardo, refiere la lógica 
alimentaria vinculada con el trabajo de autoconsumo y subsistencia:

En mi rancho, mi mamá tenía como 200 gallinas y 4 gallos, ese huevo lo comíamos y lo vendíamos... los sábados me mandaba a vender el maíz, lo cambiaba por una medida de tomate, de chile, de cebolla. (Eduardo, Guerrero, Pendular)

Los testimonios muestran la convergencia de procesos en las sociedades de autoconsumo con la incursión de modelos económicos de consumo, sobre todo, de alimentos industrializados basados en el azúcar.

Yo iba con mi papá de cacería, traíamos venado, liebre y comíamos, o vendíamos la carne. Mi papá siempre tenía maíz, frijol y cuando vendía la carne, compraba bultos de azúcar, cosas que no se perdieran. (Alma, Veracruz, Pendular)

A esto se suman formas de obtención de alimentos por cacería, recolección, cría de animales, aun entre jóvenes migrantes pendulares de recién llegada a Miguel Alemán, quienes refieren el consumo de animales de traspatio:

Allá comes un pollo, lo matas y lo comes fresco; la carne de puerco sabe más sabrosa porque el marrano recién matado. (Lucía, Guerrero, Pendular)

El habitus alimentario se conforma en relación con la variedad de alimentos propios de climas templados mientras que, al llegar a una zona árida, estos patrones de acceso y consumo se ven afectados. Los datos empíricos muestran la transición económica de la agricultura de subsistencia a la necesidad de incursionar en el trabajo asalariado en la agroindustria, lo que supone una lógica y relación distinta con los recursos naturales, porque es poco probable que lleguen a consumir los alimentos que están produciendo, comestibles inaccesibles que "no es para nosotros"(27), reproduciéndose así la distinción entre los grupos poblacionales.

\section{Habitus alimentario en el contexto de migración: prácticas y ajustes}

En esta sección comparamos los resultados entre migrantes asentados y pendulares, ya que las condiciones de posibilidad para acceder a los alimentos son distintas en cuanto al capital económico y social. Algo en común para ambos tipos de migrantes es que al insertarse en la lógica del trabajo agrícola extensivo, sus prácticas de alimentación se estructuran de acuerdo a los horarios de trabajo, las condiciones de ingesta de alimentos y el tiempo otorgado para consumirlos.

\section{Prácticas de alimentación entre migrantes asentados}

Presentamos los datos empíricos de migrantes que viven en Miguel Alemán desde hace más de cuatro años y que se trasladan de la comunidad a los campos agrícolas para trabajar. Los lonches (anglicismo de lunch) son el alimento cotidiano para esta población y es ingerido durante la jornada. Consisten en tortillas de harina de trigo enrolladas que contienen guisos con frijoles, embutidos (salchicha, jamón, chorizo) y, ocasionalmente, carne de res. Entre 10 y 12 tacos son consumidos diariamente en dos momentos: la mitad a la llegada al campo agrícola y antes de iniciar la jornada, y la otra mitad al medio día. Cada tortilla contiene un $47 \%$ de carbohidratos $^{(35)}$. Se come en las cercanías del surco, sin calentar y, de acuerdo a los testimonios, el uso de la tortilla de harina de trigo obedece a que es más fácil de enrollar que la de maíz o maseca:
Los tacos son de frijol, el huevo, la Bolo- nia [embutido con alto contenido en hari- nas y grasas de cerdo], la salchicha, carne molida, papa, atún con huevos, papas con huevo o con atún, nomás de eso y repetir. (Esperanza, Chiapas, Asentada)

Es común el consumo de estos alimentos industrializados para preparar los lonches, lo que indica una disminución de la variedad de alimentos comparado con los distintos 
vegetales, hortalizas y carnes a las que tenían acceso en el lugar de origen, por lo que tienen que "repetir". A esto sumamos el significado histórico del uso del trigo durante la conquista española y la preparación de la tortilla de harina con agua, sal y manteca propio de la zona norte del país ${ }^{(36)}$, y que forma parte de la identidad sonorense ${ }^{(37)}$. Sin embargo, esto no es asumido por toda la población migrante como parte de sus gustos, a pesar del largo tiempo de asentamiento. El consumo de la tortilla de harina de trigo obedece a una necesidad, más que a un gusto de lujo o libertad, ya que esta no forma parte del habitus alimentario, como la tortilla de maíz:

A veces me como una o dos, no me gustan, como que no me llenan, no como a gusto. (Elena, Chiapas, Asentada)

También encontramos referencias al consumo de alimentos congelados con conservadores y alimentos altos en azúcares, grasas y carbohidratos, no exentos de contradicciones en la valoración entre lo bueno y lo malo, lo accesible y lo inaccesible:

Aquí todo está embolsado, a veces ya está verde el pollo... [mis hijos] toman jugo, té, Danonino [marca comercial de yogurt] y allá no, una soda se toma a la mitad, entre dos. Les doy un pan entero, comen Sabritas [marca de papas fritas], paletas, chicles y allá no, no hay. (Mónica, Guerrero, Asentada)

Al insertarse al modelo de consumo, las prácticas de alimentación se ajustan a las condiciones materiales del lugar de asentamiento y a la disponibilidad física y económica, ya que el trabajo posibilita el acceso a recursos económicos que se invierten, en gran parte, en la alimentación. La práctica cotidiana del consumo de lonches en los campos se enfrenta a rupturas temporales y simbólicas cada fin de semana cuando, después de recibir la "raya" -salario semanal cuyo significado remonta a las haciendas mexicanas de inicios del siglo XX y el trabajo de la población campesina que, en su mayoría, era analfabeta por lo que firmaba con una raya en el listado de pagos $^{(38)}$ - se compran algunos platillos preparados, expresión de prácticas de alimentación en sociedades de consumo. Elena refiere:

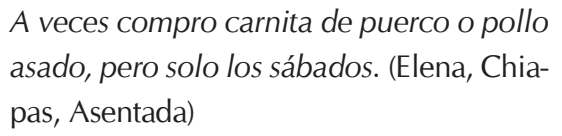

Las prácticas de alimentación se relacionan con la lógica del trabajo, la obtención de los recursos y en la definición de los fines de semana como momentos de esparcimiento y ocio, momentos que son socializados en las sociedades de consumo (endomingarse, refería Bourdieu), que se viven como la posibilidad de disfrute y distinción al asumir prácticas de alimentación basadas en el consumo, dándose por entendido que se dispone del capital económico, ya que en ese contexto "tengo que comprar todo para comer" (Mónica, Guerrero, Asentada). El aumento de las prácticas de consumo también se relaciona con la posibilidad de ingerir alimentos "naturales" o con "químicos", cuyos efectos se viven en la percepción de los padecimientos, como Angelina relata:

Aquí uno se envejece muy rápido, la piel, la comida tiene mucho químicos. Cuando voy para allá y vengo, me dicen “¿qué te hiciste?". Nada, uno allá come natural, no que aquí, todo sabe igual, el pollo, la carne. (Angelina, Chiapas, Asentada)

Encontramos una alta valoración de la dieta tradicional y los alimentos naturales percibidos como sanos, frescos y con mayor sabor. Las prácticas alimentarias transitan de la añoranza a la posibilidad de adecuar preparaciones con los ingredientes disponibles. El consumo de productos procesados se posiciona como una "elección de destino, una elección forzada, producidas por unas condiciones de existencia que [...] no deja otra opción que el gusto de lo necesario"(27), cuyos efectos se simbolizan en procesos corporales estigmatizados, como el envejecer, expresión de lo que el cuerpo digiere nutricional 
y simbólicamente, ya que es "cultura convertida en natura [...] clase hecha cuerpo"(27). Pero también se convierten en procesos de enfermedad crónica, vinculadas directamente con las prácticas de alimentación y los ajustes en la dieta, tal como Jorge refiere sobre la atención a su padre diabético:

Mi mamá hace igual para todos, porque si no él, jimagínese! nomás el caldo de pollo, cocina sin mucha grasa y las tortillas [de harina de trigo], eso sí. (Jorge, Oaxaca, Asentado)

Se muestran las contradicciones entre el consumo de alimentos por gusto (como las tortillas de harina de maíz) y el discurso medicalizado de lo que es bueno o malo comer, pero cuyas condiciones de posibilidad se obstaculizan debido a las carencias económicas. También encontramos procesos de enfermedad que de forma crónica merman la salud de la población, por lo que tratan de cambiar sus prácticas de alimentación y dejar de consumir aquellos alimentos que sociocultural y económicamente están disponibles, tal como Arnoldo refiere:

Tengo colesterol, procuro no comer tortilla de harina [de trigo], chorizo, carne de puerco; salió alto el azúcar, que no comiera pan, ni azúcar, ni refresco y por eso me estoy curando, no como cosas con grasa. (Arnoldo, Guerrero, Asentado)

Sin embargo, ponen en práctica diversas acciones para conservar su habitus alimentario frente al modelo de consumo, que pone a disposición física un alto número de productos industrializados para homogenizar los esquemas de apreciación sobre los patrones alimentarios en el mundo globalizado. Registramos prácticas de cultivo de traspatio de hierbas de olor o "aromas", como pápalo, hierba santa, hierbabuena, cilantro, así como árboles frutales, incluyendo el plátano que no es propio del desierto, pero que es altamente valorado por el uso de las hojas para la preparación de tamales. Isaura narra:
Puse hierba santa, tenía orégano, epazote. Sembraba cebolla y ajo, íbamos a comprar a un ranchito gallos y gallinas (Isaura, Veracruz, Asentada).

La siembra de "aromas" se significa como una forma de dar sazón a las comidas tradicionales ya que, de acuerdo a Vázquez ${ }^{(39)}$, son medios para materializar los recuerdos y nostalgias alimentarias, así como los saberes respecto al proceso salud/enfermedad/atención, como Arnoldo y Angelina refieren:

\begin{abstract}
A veces traemos semilla de allá, sí crecen, gentes han traído. Ese arbolito te cura los riñones. (Arnoldo, Guerrero, Asentado)
\end{abstract}

Un té de jengibre, canela y té limón, para la tos. (Angelina, Chiapas, Asentada)

Los procesos de salud-enfermedad-atención se vinculan con saberes y cultivos tradicionales que rebasan la lógica de consumo y visibilizan prácticas solidarias, que cuestionan el orden social y la medicalización de la vida cotidiana en contextos precarios. Esperanza relata:

\section{Una amiga me dio una matita de cola de caballo [planta medicinal] y le fui a dejar a una muchacha que sufre de los riñones. (Esperanza, Chiapas, Asentada)}

Estas prácticas se relacionan con la reproducción de un habitus que privilegia el uso de ciertos condimentos y alimentos, en una especie de añoranza e idealización; estas prácticas migran geográfica y simbólicamente y se significan como disposiciones a la acción, tal como Esperanza refiere:

\footnotetext{
Tengo nopales, tomates chiquitos, chayote, calabaza, mi hijo trajo semilla de chilacayote, chile y cebollín y lo sembré. (Esperanza, Chiapas, Asentada)
}

La siembra para autoconsumo les permite ahorrar y obtener alimentos sin obedecer a la lógica monetaria, como una práctica de sobrevivencia y de reproducción de saberes. 
Estas prácticas las registramos en población asentada que tienen terreno propio y aunque son pequeños (generalmente de 10 por 20 metros), han logrado cultivar en la tierra árida y polvosa, a pesar de los problemas para disponer de agua entubada.

Encontramos que las condiciones de vida como la inseguridad en la localidad también se relaciona con las prácticas de alimentación. Es común que las mujeres realicen las compras "del mandado" (alimentos) el fin de semana, cuando se ofertan bajos precios. Sin embargo, se registra el robo de alimentos tal como Elena comenta:

A una vecina entraron y le llevaron todo

el mandado. (Elena, Chiapas, Asentada)

La inseguridad ajusta las prácticas de alimentación y se diseñan cuidados para no exponer la integridad física, como evitar salir a las tiendas de abarrotes después de cierta hora a lo que se suma la impunidad de estos actos delictivos. Los datos empíricos muestran cómo las condiciones de posibilidad estructuran el habitus alimentario, que rebasan el mero acto de comer y se posicionan como una práctica cotidiana dispuesta en correspondencia con las condiciones materiales de vida, en donde la precariedad es la constante en este grupo poblacional que vive simbólica y estructuralmente distintas formas de violencia.

\section{Prácticas de alimentación entre migrantes pendulares}

Las condiciones para acceder a los alimentos entre la población que se moviliza se ajusta a las posibilidades de contar con capital económico y social durante los tránsitos migratorios. En general, los hombres solos son quienes están más expuestos al consumo de alimentos procesados y a un cambio en el tipo y momentos de la comida:

En Chiapas comes mejor, aquí ganas más bien que allá, pero no comes igual, porque en la mañana sales temprano y no te da chance de preparar el desayuno, desayunas cualquier cosa o una soda. Allá tu mamá se levanta tempano a preparar el desayuno y acá no, se sufre un poco. (Juan, Chiapas, Pendular)

Relacionado con las construcciones de género, analizamos el cambio en las prácticas de alimentación entre migrantes pendulares, principalmente hombres, que llegan a las Comunidades aledañas a los campos agrícolas y que disponen de prácticas para tener la posibilidad de contar con alguien que les prepare los alimentos, contratando el servicio de comida de mujeres de la localidad, a pesar de que los menús no sean de su gusto:

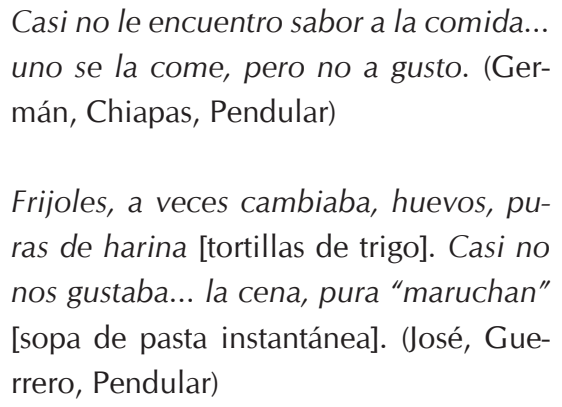

Frijoles, a veces cambiaba, huevos, puras de harina [tortillas de trigo]. Casi no nos gustaba... la cena, pura "maruchan" [sopa de pasta instantánea]. (José, Guerrero, Pendular)

La comida ofrecida por la mayoría de las mujeres consiste en los lonches antes descritos, mientras que algunas mujeres migrantes preparan ocasionalmente comidas con alimentos de sus lugares de origen cuando estos se encuentran disponibles física y económicamente. Estas prácticas pueden analizarse como estrategias de conservación, pero también como una forma de reproducir relaciones de apoyo, como refiere Germán:
Le decía al taxista o la esposa que si me podía dar lonche, se lo iba a pagar. Hay que tener confianza con la gente... no quedaba mal. (Germán, Chiapas, Pendular)

El saber culinario de las mujeres forma parte de la lógica de la mercantilización de los alimentos, contribuyendo a reproducir el habitus alimentario y cubrir las nostalgias alimentarias. Esta práctica se significa más allá de la venta de alimentos, ya que permite construir relaciones de confianza y de 
solidaridad basadas en la identidad, sobre todo, entre quienes son del mismo lugar de origen. Sin embargo, esto no exime de que las mujeres vivan abusos, como el no recibir el pago semanal de las comidas por parte de los trabajadores, quienes se movilizan de un campo a otro o de una localidad a otra.

Dentro de los campos agrícolas, la reiteración de la subordinación se expresa en la alimentación que los agroempresarios ofrecen a los trabajadores en los comedores que operan en el interior del campo. En algunos casos, la comida se da gratuitamente durante la jornada laboral y, en otros, su costo se descuenta del salario semanal. Los trabajadores alojados en los campos agrícolas consumen lo que se ofrece en el comedor sin posibilidad de decidir, ya que es un solo menú para todo el personal que, en la mayoría de los casos, es ajeno a los sabores y gustos de lo que comen en sus lugares de origen, además de padecer algunos efectos adversos en la salud, como refiere Arnoldo:

Me he enfermado de la panza, pegan cólicos con la comida, no la lavan bien. No sabe bien, no tiene sabor, como agua hervida, la sopa te la dan cruda, las tortillas contaditas, si quieres bien y si no, qué le vamos a hacer, tenemos que comprarla. (Arnoldo, Puebla, Pendular)

Las condiciones para acceder a los alimentos dentro de los campos son distintas para quienes van desde las comunidades aledañas, ya que las posibilidades de elegir qué comer se ven reducidas a lo que el comedor les ofrece o lo que pueden comprar en las tiendas del interior del campo a un alto precio. Estas prácticas resultan de una continuidad histórica y matizada del funcionamiento de las tiendas de raya en las haciendas del siglo XX, en donde los campesinos compraban productos de primera necesidad a un alto precio, por lo que se endeudaban y se veían obligados a continuar trabajando en las haciendas $^{(38)}$. Si bien esto no sucede con igual magnitud en los campos agrícolas actuales, la lógica de endeudamiento funciona como un medio para reproducir las condiciones de precariedad económica de los trabajadores agrícolas:

La comida no es igual, los frijoles bayos te los dan crudos, recalentado, huevo medio guisado, carne que te hace daño, te enfermas del estómago. Ahí mismo hay tienda, ahí mismo al dinero se le da vuelta. (Bernardo, Veracruz, Pendular)

Los efectos de la alimentación en la salud son vividos en los cuerpos de los agentes, que viven procesos de salud-enfermedad-atención en condiciones de difícil acceso a los servicios de salud o bien, con dificultades para las prácticas de autocuidado sobre todo entre los varones migrantes pendulares, entre quienes la autopercepción de la salud se relaciona con la pérdida de peso debido al cambio en las prácticas de alimentación:

Vine bien delgada del campo, aquí [en Miguel Alemán] me estoy recuperando. (Sofía, Veracruz, Pendular)

Llegué a un campo pero estaba feo, no desayunaba, estaba bien flaco. Estoy flaco todavía porque no me he alimentado bien. (Juan, Chiapas, Pendular)

Pero también hay efectos crónicos en la salud entre migrantes pendulares y se expresan en diagnósticos de diabetes e hipertensión, como Alma refiere:

Hace 6 años me dijeron que tengo diabetes, pero como todo, café, soda, tortillas, ni modo que no coma, le quitan todo a uno. (Alma, Veracruz, Pendular)

Las condiciones de posibilidad para elegir alimentos entre diabéticos trastocan esquemas sobre lo que es bueno o malo consumir, al asumir que "le quitan todo", para referirse a las escasas oportunidades de acceder a alimentos indicados. La alimentación se ajusta a las condiciones de vida, a pesar de tener conciencia de lo que puede o no comer, impidiendo configurar prácticas de cuidado, tanto en procesos salud-enfermedad-atención, como de cuidado 
en los espacios públicos, ya que los alimentos se convierten en capital altamente valorado entre población que carece de estos, ya que "te asaltan para quitarte el lonche" (Eduardo, Guerrero, Pendular).

Las prácticas de alimentación se sitúan en el campo público, en las relaciones de subordinación y violencia, la exposición a riesgos más allá del consumo de alimentos valorados como saludables o dañinos, situándose como una necesidad básica insatisfecha, expresión de la desigualdad social que se reproduce en los contextos de tránsito y asentamiento.

\section{DISCUSIÓN}

Otros estudios retoman la propuesta de Bourdieu para analizar la alimentación ${ }^{(26,40,41,42)}$, y consideran la clase y posición social ${ }^{(43)}$, así como las condiciones del trabajo ${ }^{(44)}$. Los datos empíricos muestran la red de relaciones vinculadas con el trabajo agrícola, la migración y la lógica de consumo, que estructuran la práctica alimentaria predispuesta a elecciones por necesidad, no por gusto.

Díaz y García ${ }^{(45)}$, Lupton ${ }^{(46)}$ y Tomlinson ${ }^{(47)}$ refieren que las elecciones y prácticas alimentarias se relacionan con el grupo social de pertenencia y la distinción. De igual forma, Florence Théodore Rowlerson ${ }^{(48)}$ utiliza el concepto de habitus alimentario para referir que las elecciones y prácticas alimentarias no son individuales, sino que son interiorizadas en el proceso de socialización. En esta investigación encontramos que el habitus alimentario se reconfigura al migrar, pero los agentes no son pasivos frente al modelo alimentario. Más allá de aceptar la comida del lugar de recepción, los gustos culinarios se reproducen y construyen formas de mantener la identidad en los contextos de migración, mientras que se asumen otras prácticas para encajar con la cultura dominante ${ }^{(44)}$.

Mata Codesal(49) analiza las prácticas de migrantes para reproducir y conservar la identidad culinaria, lo que también encontramos en esta investigación entre migrantes asentados como el cultivo de traspatio, la cría de animales y la venta de comida propia de los lugares de origen, lo que además posibilita relaciones de solidaridad y cohesión social. Otra de las prácticas documentada por Alcántara ${ }^{(24)}$ es la preparación de tortillas combinando harina de trigo y de maíz, analizada como práctica de conservación de los gustos y del habitus alimentario frente a los patrones modernizadores de la alimentación.

Da Morais et al. ${ }^{(50)}$ refieren que el habitus permite comprender las relaciones entre la comida, el ambiente físico, las inequidades en salud y las desigualdades de género. En la investigación encontramos una red de relaciones entre las condiciones y la dinámica del trabajo agrícola, el ambiente geográfico (migrar de zonas templadas y húmedas a una desértica), la oferta comercial de alimentos y las disposiciones de género, que estructuran las prácticas alimentarias. En otra publicación analizamos las relaciones de género y el proceso alimentario que, si bien muestra la participación de los varones en prácticas como realizar las compras de alimentos y lavar los utensilios de cocina, consideran la tarea alimentaria como propia de las mujeres, reproduciéndose las normas hegemónicas de género ${ }^{(18)}$. Actividades como el cultivo y cría de traspatio son realizadas por mujeres, quienes tienen dobles o triples jornadas de trabajo, al combinar las labores del campo con la elaboración de alimentos para la venta y la crianza de los hijos y las hijas, reproduciéndose la división sexual y social del trabajo que se significan en sus vidas como mayor tiempo dedicado al trabajo doméstico no remunerado e invisibilizado frente al sistema económico, pero que permiten su reproducción ${ }^{(51,52,53)}$.

Los resultados de investigaciones ${ }^{(6,24,54)}$ y los datos cualitativos encontrados aquí, muestran los riesgos para la salud entre trabajadores agrícolas relacionados con las prácticas de alimentación, que van desde la desnutrición hasta la obesidad y diabetes. El consumo de alimentos industrializados significa mejora económica y social, una forma de lograr la modernidad ${ }^{(55,56,57)}$, pero entre la población jornalera en México se reportan limitaciones económicas para satisfacer 
las necesidades alimenticias ${ }^{(58)}$. En los datos empíricos encontramos un aumento en el consumo de productos industrializados disponibles física y económicamente en el lugar de recepción, mientras que se da una nostalgia alimentaria por alimentos frescos, percibidos como más sanos en comparación con los procesados. Sin embargo, es necesario considerar el discurso médico sobre los esquemas de percepción y apreciación de alimentos naturales y alimentos industrializados, como el azúcar.

De acuerdo con Mintz ${ }^{(59)}$, la producción de azúcar sirvió para cubrir las necesidades energéticas de los trabajadores en las sociedades industrializadas, lo que muestra la relación entre estructuras económicas y prácticas de alimentación y, si bien son una acción individual, responden a esquemas de percepción y apreciación ajustadas a las estructuras que las producen. Encontramos que, desde las localidades de origen, las y los trabajadores aprenden y asimilan nuevas disposiciones para la acción, como el consumo de alimentos procesados como el azúcar, que se significa como un bien obtenido con la venta de la cosecha. Esto es resultado de patrones globalizadores de la industria alimentaria, que se refuerzan al insertarse en sociedades de consumo, por lo que la ingesta de algunos alimentos procesados aumenta y forma parte de las prácticas, aun cuando muchos de ellos no son del gusto y preferencia, lo que reconfigura el habitus alimentario en el contexto migratorio.

\section{CONCLUSIONES}

De acuerdo al objetivo de analizar y comparar el habitus alimentario y el cambio en las prácticas alimentarias entre trabajadores agrícolas migrantes pendulares y asentados, encontramos evidencia empírica sobre el ajuste del habitus a las lógicas del lugar de recepción y asentamiento. Analizar el habitus permite comprender las prácticas como procesos complejos dentro de un sistema de relaciones, en el cual los agentes ocupan distintas posiciones en el campo social. En el caso de trabajadores agrícolas, las condiciones precarias de migrar y asentarse, la inestabilidad laboral y los obstáculos para acceder a servicios sociales y de salud, son condiciones que posibilitan ciertas prácticas alimenticias.

Analizamos las lógicas de las prácticas ajustadas por condiciones económicas y la disponibilidad de alimentos. A pesar de que estos agentes cosechan alimentos, no son los consumidores finales, sino que se trata de productos de exportación, de distinción. Su mano de obra forma parte de la cadena de producción globalizada; primordial pero subordinada material y simbólicamente debido a la imposibilidad de consumir estos productos que son para "otros", mecanismo que reproduce las relaciones de dominación. Si bien los migrantes asentados cuentan con mayor capital social y económico para obtener alimentos, esto no los exime de dificultades para acceder a ellos, relacionado con la precariedad económica y la inaccesibilidad física de alimentos que son del gusto, condiciones que constriñen material y simbólicamente la práctica alimentaria.

Los testimonios muestran el cambio sociohistórico de modelos económicos en transición, de las localidades de origen a las de migración, y que se acotan a procesos como la alimentación. Evidenciamos que la lógica del trabajo agrícola, como estructura estructurante, ajusta y posibilita el tipo de alimento que se come en el surco, los momentos de la comida y los problemas que van desde el acceso físico y económico hasta la inseguridad traducida en robo de alimentos, expresión de una necesidad básica insatisfecha y de la violencia estructural que vive la población.

El habitus alimentario muestra la relación entre economías que transitan de la subsistencia y autoconsumo hacia economías monetarizadas, y si bien pueden disponer de dinero por su trabajo en la agroindustria gran parte se destina a la compra de alimentos. Encontramos que la variedad de alimentos de origen vegetal y animal se reduce en el lugar de asentamiento, mientras se añoran los alimentos frescos del lugar de origen. Sin embargo, 
también se evidencia que alimentos procesados y azucarados ya formaban parte de las prácticas en sus lugares de origen, aunque las posibilidades de acceso a ellos eran más limitados geográfica y económicamente.

El consumo de alimentos baratos y de alta densidad energética se vincula con el sector trabajador de baja remuneración y se significa como un medio de diferenciación con los grupos dominantes; estas prácticas responden a condiciones objetivas de existencia para las y los trabajadores agrícolas en contextos de migración, como expresión de "la necesidad de reproducir al menor coste la fuerza de trabajo"(27); de allí el objetivo de analizar cómo se estructura esta práctica con procesos macroeconómicos y socioculturales.

Registramos el cultivo de traspatio, la cría de animales, el intercambio de semillas y la venta de comidas tradicionales como prácticas de sobrevivencia entre la población asentada. Tales estrategias son puestas en práctica, principalmente, por mujeres, quienes obtienen ingresos económicos con estas actividades. Si bien estas actividades se relacionan con las prácticas socialmente atribuidas a las mujeres, les permite tener cierta autonomía económica. Los migrantes pendulares, por su movilidad constante y el limitado acceso a los insumos y enseres necesarios para la preparación de alimentos, ven disminuidas las posibilidades de elegir qué comer. Para los migrantes pendulares varones, estas posibilidades son aún más escasas: debido a prácticas relacionadas con las construcciones sociales de género, su consumo de alimentos se da por necesidad y lo que el mercado les oferta. Algunos de ellos, los más jóvenes reportan una pérdida de peso por disminuir las ingestas. Sin embargo, esta situación nutricional permanece invisibilizada por ellos mismos y por el sistema de salud, cuyos efectos a largo plazo habrá que analizar. Los migrantes asentados refieren enfermedades crónicas, lo que indica el espectro de posibilidades de enfermar en esta población, que se complejiza por la movilidad y las dificultades de acceso a los servicios de salud. Los tipos de migración se relacionan con las condiciones que constriñen la práctica alimentaria, por lo que analizar el habitus alimentario en relación con tales condiciones permite comprender las desigualdades que merman la vida cotidiana y la salud de la población trabajadora agrícola.

Concluimos que las prácticas de alimentación entre las y los trabajadores agrícolas se estructuran como una práctica por necesidad, al ver reducidas sus posibilidades de elección basadas en el gusto por los alimentos. Este mecanismo reproduce material y simbólicamente la posición subordinada de las y los trabajadores frente al modelo alimentario y económico globalizado y los efectos en la salud de estas prácticas alimentarias. Los datos empíricos contribuyen como evidencia al trazar posibles caminos para lograr el desarrollo sostenible, justo y equitativo, comprendiendo el sentido lógico de las prácticas alimentarias en relación con las condiciones precarias de vida y las dificultades de atención a la salud entre trabajadores agrícolas. Por último, ubicar la localidad como zona de atención prioritaria del programa Cruzada contra el Hambre, indica que la alimentación es un tema crucial, por lo que habrá que analizar posteriormente la relación entre las acciones del programa y los efectos en la salud de la población.

\section{AGRADECIMIENTOS}

Se agradece al Consejo Nacional de Ciencia y Tecnología (CONACYT) de México el apoyo económico para la realización de esta investigación como parte de los estudios doctorales de la primera autora, así como al Colegio de la
Frontera Sur, institución de adscripción. El apoyo económico fue aprobado para el periodo enero 2015-diciembre 2018, tiempo en el cual se realizó la investigación titulada "Relaciones sociales y prácticas de alimentación de migrantes del sureste mexicano residentes en una comunidad agroindustrial de Sonora". 


\section{REFERENCIAS BIBLIOGRÁFICAS}

1. Organización de las Naciones Unidas. Objetivos y metas del desarrollo sostenible [Internet]. 2018 [citado 31 mar 2018]. Disponible en: https:// tinyurl.com/y8d2cd74.

2. Naciones Unidas. Objetivos de desarrollo del milenio: Informe de 2015 [Internet]. New York: NU; 2015 [citado 28 jun 2019]. Disponible en: https://tinyurl.com/yyymvnkn.

3. Consejo Nacional de Población (CONAPO). Datos abiertos del índice de marginación [Internet]. 2016 [citado 12 abr 2018]. Disponible en: https://tinyurl.com/yyozejgt.

4. De Grammont H, Lara S. Encuesta a hogares de jornaleros migrantes en regiones hortícolas de México: Sinaloa, Sonora, Baja California Sur y Jalisco. México: UNAM; 2004.

5. Tlachinollan, Centro de Derechos Humanos de la Montaña. La montaña de Guerrero: tierra de mujeres migrantes [Internet]. México: Tlachinollan; 2011 [citado 12 abr 2018]. Disponible en: https://tinyurl.com/y5vybw9x.

6. Castañeda J. Inseguridad alimentaria y obesidad en jornaleros agrícolas migrantes del estado de Sonora. [Tesis de Maestría]. Hermosillo: CIAD; 2017.

7. Ortega I, Alcalá G. Pobreza, migración y seguridad alimentaria. En: Sandoval S, Meléndez J. Cultura y seguridad alimentaria: Enfoques conceptuales, contexto global y experiencias locales. México: Plaza y Valdés; 2008.

8. Ortega I, Castañeda A, Sariego J. Los jornaleros agrícolas invisibles productores de riqueza: Nuevos procesos migratorios en el noroeste de México. México: CIAD, Fundación Ford, Plaza y Valdés; 2007.

9. Aranda P, Castro C. El campo de la agroindustria en el noroeste de México y la salud de sus jornaleras: una propuesta de estudio. Salud Colectiva. 2016;12(1):55-70. doi: https://doi.org/10.18294/ sc. 2016.878 .

10. Arellano C, Castro C, Aranda P. Del favor al derecho: condiciones de posibilidad para el acceso a la atención de la salud de trabajadoras agrícolas en el Noroeste de México. En: Freyermuth G. El derecho a la protección de la salud de las mujeres indígenas en México, análisis nacional y de casos desde una perspectiva de Derechos Humanos [Internet]. México: CNDH; 2017 [citado 31 mar 2018]. Disponible en: https://tinyurl.com/ y6tpq519.
11. Valdivia M, Sánchez L. Protección laboral para los jornaleros agrícolas en México. Apuntes para la Equidad. 2017;(3):1-4.

12. Rivera F. La provisión de seguridad social a jornaleros agrícolas en México: el caso de las modificaciones a la Ley del Seguro Social en los años 1995 y 2005. [Tesis de maestría]. México: FLACSO; 2005.

13. Hernández M, Gutiérrez $P$, Reynoso N. Diabetes mellitus en México: El estado de la epidemia. Salud Pública de México. 2013;55(Suppl 2):S129-S136.

14. Bello-Chavolla OY, Rojas-Martinez R, AguilarSalinas CA, Hernández-Avila M. Epidemiology of diabetes mellitus in México. Nutrition Reviews. 2017;75(1):4-12. doi: 10.1093/nutrit/nuw030.

15. World Health Organization. Quality of care is key to tackling Mexico's diabetes emergency. Bulletin of World Health Organization. 2017;95(6):393394. doi: 10.2471/BLT.17.020617.

16. Bourdieu P. El sentido práctico. Buenos Aires: Siglo XXI Editores; 2007.

17. Bourdieu $P$, Wacquant L. Una invitación a la sociología reflexiva. Buenos Aires: Siglo XXI Editores; 2005.

18. Arellano M, Alvarez G, Tuñón E, Huicochea L. El trabajo de alimentar: proceso alimentario entre trabajadores y trabajadoras agrícolas migrantes en Miguel Alemán, Sonora. Revista Interdisciplinaria de Estudios de Género. 2018;(4):1-35. doi: 10.24201/eg.v4i0.240.

19. Gobierno del estado de Sonora, Ayuntamiento de Hermosillo. Programa de Desarrollo Urbano del Centro de Población Miguel Alemán [Internet]. 2016 [citado 5 abr 2018]. Disponible en: https:// tinyurl.com/y5fz7nj2.

20. Bracamonte Sierra A, Valle Dessens N, Méndez Barrón R. La nueva agricultura sonorense: historia reciente de un viejo negocio. Región y Sociedad. 2007; 19:51-70.

21. Gobierno del Estado de Sonora, Secretaría de Agricultura, Ganadería, Recursos Hidráulicos, Pesca y Acuacultura. Programa de mediano plazo agrícola 2016-2021 [Internet]. 2016 [citado 5 abr 2018]. Disponible en: https://tinyurl.com/yx9xwwpn.

22. Gobierno de México, Secretaría de Desarrollo Social. Lineamientos de organización y funcionamiento de los comités comunitarios de la Cruzada Contra el Hambre y de los Programas Sociales Federales [Internet]. 2015 [citado 5 oct 2017]. 
Disponible en: http://tinyurl.com/https-www-gobmx-cms-uploads.

23. Ortega I, Rosales C, Guernsey J, Aranda P, Castañeda A, Saucedo S, Montaño C, Contreras A. Migration, agribusiness and nutritional status of children under five in Northwest Mexico. International Journal of Environmental Research and Public Health. 2012;9(1):33-43. doi: 10.3390/ ijerph9010033.

24. Alcántara Y. "A todo se acostumbra uno, menos a no comer... azúcar": Prácticas de alimentación ante la diabetes en grupos domésticos triquis, inmigrantes en Sonora. [Tesis de Maestría]. Hermosillo: COLSON; 2014.

25. Farmer P. On suffering and structural violence: A view from below. Daedalus. 1996;125(1):261-283.

26. Martín E. Conflicto entre lo sano: un estudio sociológico de la alimentación en clases populares en Andalucía. Sevilla: Consejería de Salud; 2005.

27. Bourdieu P. La distinción: criterio y bases sociales del gusto. México: Taurus; 2006.

28. Gobierno de México, Secretaría de Desarrollo Social. Diagnóstico del Programa de Atención a Jornaleros Agrícolas. México: Secretaría de Desarrollo Social; 2010.

29. Rojas T. La crisis del sector rural y el coste migratorio en México. Iberoforum. 2009;4(8):40-81.

30. Galindo J. Encuentro de subjetividades, objetividad descubierta: La entrevista como centro de trabajo etnográfico. Estudios sobre las Culturas Contemporáneas. 1987;1(3):151-183.

31. Mayan M. Una introducción a los métodos cualitativos: Módulo de entrenamiento para estudiantes y profesionales. Canadá: Qualitative Institute Press; 2001.

32. Taylor S, Bogdan R. Introducción a los métodos cualitativos de investigación: La búsqueda de significados. Barcelona: Paidós Básica; 1994.

33. Valles M. Técnicas cualitativas de investigación social. Madrid: Síntesis Sociología; 2000.

34. Zizumbo D, Flores A, Colunga P. The food system during the formative period in West Mesoamerica. Economic Botany. 2014;68(1):67-84.

35. Zazueta M. Estado nutricional de calcio en escolares hermosillenses con base en su ingestión dietaria y excreción urinaria. [Tesis de maestría]. Hermosillo: CIAD; 2015.
36. Díaz B. Historia verdadera de la conquista de la Nueva España. Madrid: Historia; 1984.

37. Sandoval S, Camarena D. Consumo de alimentos de la población sonorense: tradición versus internacionalización. Estudios Sociales. 2012;(2):53-72.

38. Jarquín $M$, Leal J, Luna $P$, Rendón $R$, Romero $M$. Origen y evolución de la hacienda en México: siglos XVI al XX. Estado de México: Colegio Mexiquense, Universidad Iberoamericana, INAH; 1990.

39. Vázquez J. De la nostalgia culinaria a la identidad alimentaria transmigratoria: la preparación de alimentos en restaurantes mexicanos en Estados Unidos. [Tesis de doctorado]. Barcelona: Universitat de Barcelona; 2015.

40. Sato PM, Gittelsohn J, Unsain RF, Roble OJ, Scagliusi FB. The use of Pierre Bourdieu' s distinction concepts in scientific articles studying food and eating: A narrative review. Appetite. 2016;96:174186. doi: 10.1016/j.appet.2015.09.010.

41. Willekens $M$, Lievens J. Boundary tastes at work: The gendered effect of authority positions in the workplace on taste in clothing and food. Sociological Perspectives. 2015;58(1):78-96. doi: $10.1177 / 0731121414556545$.

42. Bava CM, Jaeger SR, Park J. Constraints upon food provisioning practices in 'busy' women's lives: Trade-offs which demand convenience. Appetite. 2008;50:486-498. doi: 10.1016/j.appet.2007. 10.005 .

43. Backett-Milburn KC, Wills WJ, Roberts ML, Lawton J. Food, eating and taste: parents' perspectives on the making of the middle class teenager. Social Science \& Medicine. 2010;71(7):13161323. doi: 10.1016/j.socscimed.2010.06.021.

44. Woodhall-Melnik J, Matheson FI. More than convenience: the role of habitus in understanding the food choices of fast food workers. Work Employment \& Society. 2017;31(5):800-815. doi: 10.1177/0950017016648255.

45. Díaz C, García I. La mirada sociológica hacia la alimentación: análisis crítico del desarrollo de la investigación en el campo alimentario. Política y Sociedad. 2014;51(1):15-49.

46. Lupton D. The hearth of the meal: food preferences and habits among rural Australian couples. Sociology of Health and Illness. 2000;22(1):94 109. doi: 10.1111/1467-9566.00193.

47. Tomlinson M. Lifestyle and Social Class. European Sociological Review. 2003;19(1):97-111. doi: 10.1093/esr/19.1.97. 
48. Rowlerson FT. Marco teórico y conceptual del insumo socio-cultural de la canasta normativa [Internet]. México: Evalúa DF, Consejo de Evaluación del Desarrollo Social del Distrito Federal; 2010 [citado 4 abr 2018]. Disponible en: https:// tinyurl.com/yyacbvxo.

49. Mata Codesal D. Eating abroad, remembering (at) home. Anthropology of Food. 2010;7.

50. de Morais Sato $P$, da Rocha Pereira $P$, de Carvalho Stelmo I, Unsain RF, Ulian MD, Sabatini F, Martins PA, Scagliusi FB. Eating pactices and habitus in mothers: A Brazilian population-based survey. Appetite. 2014;82:16-28. doi: 10.1016/j. appet.2014.07.002.

51. Brunet I, Santamaría C. La economía feminista y la división sexual del trabajo. Culturales. 2016;4(1):61-86.

52. Platt L, Polavieja J. Saying and doing gender: intergenerational transmission of attitudes toward the sexual division of labour. European Sociological Review. 2016;32(6):820-834. doi: 10.1093/ esr/jcw037.

53. Vizcarra I. Entre las desigualdades de género: un lugar para las mujeres pobres en la seguridad alimentaria y el combate al hambre. Argumentos. 2008;21(57):141-173.

54. Haro J. Globalización y salud de los trabajadores: Jornaleros agrícolas y producción de uva en Pesqueira, Sonora. Región y Sociedad. 2007;19(40):73-105.

55. Bertran M. Acercamiento antropológico de la alimentación y salud en México. Physis: Revista de Saúde Coletiva. 2010;20(2):387-411.

56. Alvarez G, Eroza E, Ramírez C. Diagnóstico sociocultural de la alimentación de los jóvenes en Comitán, Chiapas. Medicina Social. 2009;4(1):35-51.

57. Tuñón-Pablos E, Debry J. Risk factor for overweight and obesity among Mexican children in New York. International Journal of Population Research. 2016;1-8. doi: 10.1155/2016/2420167.

58. Posadas F. Mercado de trabajo de los jornaleros agrícolas en México. Región y Sociedad. 2018;30(72):1-25.

59. Mintz S. Dulzura y poder: el lugar del azúcar en la historia moderna. México: Siglo XXI Editores; 1996.

FORMA DE CITAR

Arellano Gálvez MC, Alvarez Gordillo GC, Eroza Solana E, Huicochea Gómez L, Tuñón Pablos E. Habitus alimentario: prácticas entre trabajadores agrícolas migrantes en una comunidad de Sonora, México. Salud Colectiva. 2019;15:e1843. doi: 10.18294/sc.2019.1843.

Recibido: 19 de abril de 2018 | Versión final: 18 de abril de 2019 | Aprobado: 14 de junio de 2019

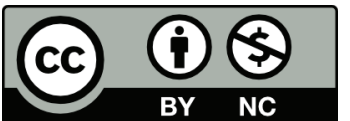

Esta obra está bajo una licencia de Creative Commons Reconocimiento-NoComercial 4.0 Internacional. Reconocimiento - Permite copiar, distribuir y comunicar públicamente la obra. A cambio, se debe reconocer y citar al autor original. No Comercial - Esta obra no puede ser utilizada con finalidades comerciales, a menos que se obtenga el permiso. 